\title{
Consumer willingness to pay for green food in Malaysia
}

\begin{abstract}
The purpose of this research is to identify how much consumers are willing to pay for green foods in Malaysia. A survey was conducted in Peninsular Malaysia of 1,355 respondents in order to explore their willingness to pay for green food products. Contingent valuation method was used to determine consumers' willingness to pay for green foods. Theory of planned behavior was expended in this research to find the willingness to pay for green foods among consumers in Malaysia. The results indicate that gender, geographical area, income, consumers' preference, motivation, intention, perception, environmental friendliness, and food safety significantly influence consumers' willingness to pay for green foods.
\end{abstract}

Keyword: Binary logistic model; Contingent valuation method; Green foods; Malaysian consumers; Theory of planned behavior; Willingness to pay 